\title{
Localization on Certain Graphs with Strongly Correlated Disorder
}

\author{
Sthitadhi Roy $\oplus^{1,2, *}$ and David E. Logan $\oplus^{1,3, \dagger}$ \\ ${ }^{1}$ Physical and Theoretical Chemistry, Oxford University, South Parks Road, Oxford OX1 3QZ, United Kingdom \\ ${ }^{2}$ Rudolf Peierls Centre for Theoretical Physics, Clarendon Laboratory, Oxford University, \\ Parks Road, Oxford OX1 3PU, United Kingdom \\ ${ }^{3}$ Department of Physics, Indian Institute of Science, Bangalore 560012, India
}

(Received 29 July 2020; accepted 17 November 2020; published 15 December 2020)

\begin{abstract}
Many-body localization in interacting quantum systems can be cast as a disordered hopping problem on the underlying Fock-space graph. A crucial feature of the effective Fock-space disorder is that the Fockspace site energies are strongly correlated - maximally so for sites separated by a finite distance on the graph. Motivated by this, and to understand the effect of such correlations more fundamentally, we study Anderson localization on Cayley trees and random regular graphs, with maximally correlated disorder. Since such correlations suppress short distance fluctuations in the disorder potential, one might naively suppose they disfavor localization. We find however that there exists an Anderson transition, and indeed that localization is more robust in the sense that the critical disorder scales with graph connectivity $K$ as $\sqrt{K}$, in marked contrast to $K \ln K$ in the uncorrelated case. This scaling is argued to be intimately connected to the stability of many-body localization. Our analysis centers on an exact recursive formulation for the local propagators as well as a self-consistent mean-field theory; with results corroborated using exact diagonalization.
\end{abstract}

DOI: 10.1103/PhysRevLett.125.250402

Disorder-induced localization of noninteracting quantum particles - the phenomenon of Anderson localization (AL) - has been one of the most profound discoveries in physics [1]. Its robustness to interactions in quantum many-body systems has lately been a major research theme, under the banner of many-body localization (MBL) [2-5] (see Refs. [6-8] for reviews and further references). MBL systems fall outside the paradigm of conventional statistical mechanics allowing for novel quantum phases, and are thus of fundamental interest.

Efforts to understand the MBL phase and the accompanying MBL transition have ranged from extensive numerical studies $[7,9,10]$ and phenomenological treatments [11-16] to studying the problem directly on the Fock space [17-27]. One virtue of the latter is that the problem can be cast as a disordered hopping problem on the Fock-space graph, thus offering the prospect of exploiting techniques and understandings developed for AL. However, MBL on Fock space is fundamentally different from conventional $\mathrm{AL}$ on high-dimensional graphs, due to the presence of maximal correlations in the effective Fock-space disorder: the statistical correlation between two Fock-space site energies, scaled by their variance, approaches its maximum value of unity in the thermodynamic limit, for any pair separated by a finite Hamming distance on the Fock-space graph. This was found to be a necessary condition for MBL to exist [27].

Motivated by this, here we ask a fundamental question: what is the fate of $\mathrm{AL}$ on random graphs with maximally correlated disorder? In parallel to the case of Fock-space disorder, the correlation between the disordered site energies of any two sites separated by a finite distance on the graph takes its maximum value in the thermodynamic limit. In suppressing fluctuations in the site energies, one might naively suppose these correlations would strongly favor delocalization; indeed it is not a priori obvious that a localized phase must exist in such a case. Nevertheless, not only do we find inexorably a localized phase and an Anderson transition, but also that the scaling of the critical disorder with graph connectivity is qualitatively different to that for the standard model with uncorrelated disorder. These models thus introduce a novel class of AL problems with intimate connections to the problem of MBL on Fock space, qualitatively distinct from $\mathrm{AL}$ problems with nonmaximally correlated disorder [28-31].

Concretely, we consider a disordered tight-binding model on a rooted Cayley tree [as well as on random regular graphs (RRGs) which are locally treelike]. For uncorrelated disorder, such models have served as archetypes for studying a range of phenomena such as localization transitions, multifractality, and glassy dynamics on complex high-dimensional graphs [32-48]. The model Hamiltonian is

$$
H=\Gamma \sum_{\langle i, j\rangle}[|i\rangle\langle j|+\text { H.c. }]+W \sum_{i} \epsilon_{i}|i\rangle\langle i|
$$

in the position basis $\{|i\rangle\}$, where $\langle i, j\rangle$ denotes a sum over nearest neighbour pairs. We denote the branching number 
of the tree by $K$ and the total number of generations in a finite-sized tree by $L$; the total number of sites in the tree is $N \sim K^{L}$. The set of correlated random site energies, $\left\{\epsilon_{i}\right\}$, is fully specified by an $N$-dimensional joint distribution. To mimic the case of many-body systems on Fock space $[21,27,49]$, we take these distributions to be multivariate Gaussians, $\mathcal{N}(\mathbf{0}, \mathbf{C})$, characterized completely by the covariance matrix $\mathbf{C}$ [27]. Taking a cue from disordered interacting local Hamiltonians, we consider the matrix elements $C_{i j}$ to depend only on the distance $\ell_{i j}$ between a pair of sites. To impose the maximally correlated limit, we consider

$$
C_{i j}=\left\langle\epsilon_{i} \epsilon_{j}\right\rangle=f\left(\ell_{i j} / L\right) ; \quad \lim _{x \rightarrow 0} f(x)=1 .
$$

The functional form of $f$ does not qualitatively affect our results, but for concreteness in numerical calculations we take $C_{i j}=\exp \left[-\ell_{i j} / \lambda L\right]$ with $\lambda=1$ [50]. The choice of the argument of $f$ is motivated by the form of correlations in the Fock-space disorder of disordered many-body systems; for $p$-local Hamiltonians the analogous $f$ was shown to be a $p$ th-order polynomial of $\ell_{i j} / \ln N_{\mathcal{H}}, N_{\mathcal{H}}$ being the Fockspace dimension [27].

Our analysis centers on the local Feenberg self-energy $S_{i}(\omega) \equiv X_{i}(\omega)-i \Delta_{i}(\omega)$, defined via the local propagator as $G_{i}(\omega)=\left[\omega^{+}-\epsilon_{i}-S_{i}(\omega)\right]^{-1}$ with $\omega^{+}=\omega+$ in $\left(\eta=0^{+}\right)$. We focus on the imaginary part of the self-energy, $\Delta_{i}(\omega)$, as it serves as a probabilistic order parameter for a localization transition. Physically, $\Delta_{i}(\omega)$ gives the rate of loss of probability from site $i$ into states of energy $\omega$. In a delocalized phase $\Delta_{i}(\omega)$ is finite, whereas in a localized phase it vanishes $\propto \eta$ [with $y_{i}(\omega)=\Delta_{i}(\omega) / \eta$ finite], both with unit probability. These characteristics of $\Delta_{i}(\omega)$ have long been used successfully to understand Anderson transitions [1,32,52-55]; and, more recently, MBL transitions on Fock space [21,24,27].

We focus on the self-energy of the root site $(i=0)$ of the rooted Cayley tree. $S_{0}(\omega)$ is given exactly by

$$
S_{0}(\omega)=\Gamma^{2} \sum_{i_{1} \in \mathbb{N}[0]}\left[\omega^{+}-W \epsilon_{i_{1}}-S_{i_{1}}^{(0)}\right]^{-1},
$$

with the sum over all sites in the first generation, and $S_{i_{1}}^{(0)}$ the self-energy of site $i_{1}$ with the root site removed (with $\mathrm{N}\left[i_{n}\right]$ denoting the set of neighbours of $i_{n}$ on generation $n+1)$. One could in principle now approximate the self-energy on the right-hand side of Eq. (3) by a typical $S_{\text {typ }}$, and obtain the distribution of $S_{0}$ self-consistently $[21,24,27]$. Here however we go far beyond such a treatment, addressing Eq. (3) to arbitrarily high orders via an exact recursive method. We first sketch the formulation, focusing on the localized phase, in particular its stability and self-consistency; whence the quantity of interest is $y_{0}(\omega)$.
From Eq. (3), $y_{0}(\omega)$ can be expressed as

$$
y_{0}=\sum_{i_{1} \in \mathrm{N}[0]} \frac{\Gamma^{2}}{\Omega_{i_{1}}^{2}}\left[1+y_{i_{1}}^{(0)}\right] ; \quad \Omega_{i_{1}}=\omega-W \epsilon_{i_{1}}-X_{i_{1}}^{(0)} .
$$

This is a recursion relation, which can be iterated as

$y_{0}=\sum_{i_{1} \in \mathrm{N}[0]} \frac{\Gamma^{2}}{\Omega_{i_{1}}^{2}}\left[1+\sum_{i_{2} \in \mathrm{N}\left[i_{1}\right]} \frac{\Gamma^{2}}{\Omega_{i_{2}}^{2}}\left[1+\sum_{i_{3} \in \mathrm{N}\left[i_{2}\right]} \frac{\Gamma^{2}}{\Omega_{i_{3}}^{2}}[1+\cdots\right.\right.$.

In Eq. (5), for any site $i_{n}$ on generation $n$ of the tree, $\Omega_{i_{n}}=\omega-W \epsilon_{i_{n}}-X_{i_{n}}^{\left(i_{n-1}\right)}(\omega)$, with $X_{i_{n}}^{\left(i_{n-1}\right)}$ the real part of the self-energy of site $i_{n}$ with its (unique) neighbor $i_{n-1}$ on the previous generation removed. As for the imaginary part of the self-energy, a recursion relation for the real part can also be derived from Eq. (3). This leads to a recursion relation for $\Omega_{i_{n}}$,

$$
\Omega_{i_{n}}=\omega-W \epsilon_{i_{n}}-\sum_{i_{n+1} \in \mathrm{N}\left[i_{n}\right]} \frac{\Gamma^{2}}{\Omega_{i_{n+1}}^{2}},
$$

with the boundary condition $\Omega_{i_{L}}=\omega-W \epsilon_{i_{L}}$ for a tree with $L$ generations. Equations (5) and (6) comprise the complete set of recursion relations required to compute $y_{0}(\omega)$ to all orders. We now make key conceptual points about the stability of the localized phase or lack thereof, and describe our results.

Note that by evaluating $y_{0}(\omega)$ using Eq. (5) for many disorder realizations, one can generate its entire distribution $P_{y_{0}}$, and also compute its typical value via $\ln y_{0, \text { typ }}=\int d y_{0} P_{y_{0}}\left(y_{0}\right) \ln y_{0}$. A stable localized phase is indicated by $y_{0, \text { typ }}$ taking a finite value independent of system size; whereas the delocalized phase is identified via a systematic growth of $y_{0, t y p}$ with system size, such that it diverges in the thermodynamic limit. The disorder strength separating these two behaviors, if present, is the critical disorder. Numerical results for the localization phase diagram so obtained for a $K=2$ Cayley tree with maximally correlated disorder are shown in Fig. 1. Considering the band center $\omega=0$ as an example [panel (a)], $\ln y_{0, \text { typ }}$ is independent of $L$ for $W>W_{c}$ whereas it diverges with $L$ for $W<W_{c}$; thus showing that a localization transition is indeed present in the model. The phase diagram similarly obtained in the entire $\omega-W$ plane is given in Fig. 1(b), which shows the presence of mobility edges in the spectrum. Finally, in Fig. 1(c), the distribution of $y_{0}$ is shown for a representative disorder in the localized phase, and shows excellent agreement with a Lévy distribution characteristic of a localized phase, $P_{y_{0}}\left(y_{0}\right)=$ $\sqrt{\kappa / \pi} y_{0}^{-3 / 2} e^{-\kappa / y_{0}}$ with scale parameter $\kappa$.

The stability of the localized phase can also be understood as the convergence of the recursion relation in Eq. (5). The series for $y_{0}$ can be organized as 

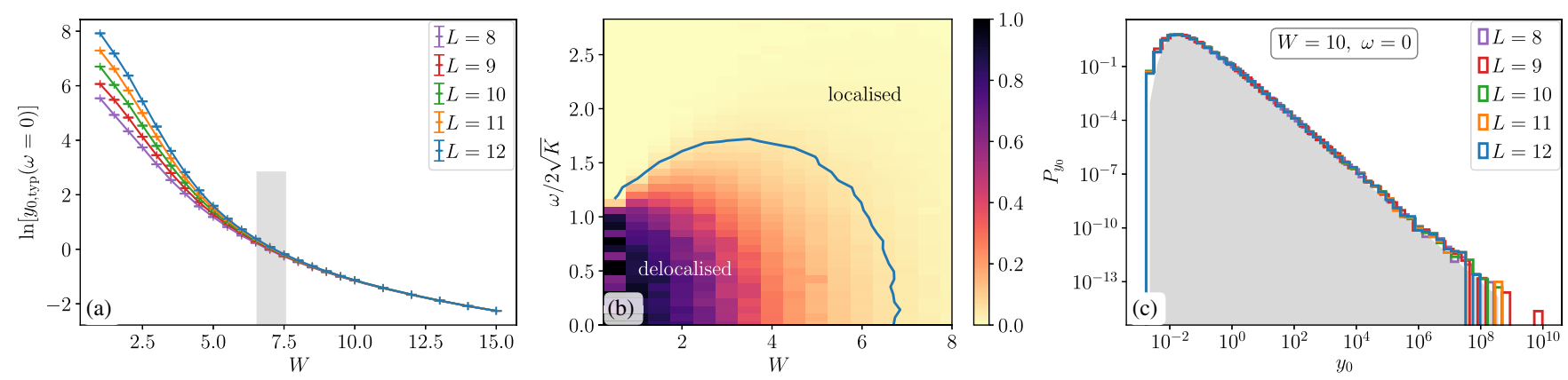

FIG. 1. For a $K=2$ rooted Cayley tree, numerical results from the exact recursion method. (a) The typical $y_{0, \text { typ }}$ at $\omega=0$, vs disorder strength $W$ (with $\Gamma \equiv 1$ ) for different total generation numbers $L$. For $W>W_{c}, y_{0, \text { typ }}$ is independent of $L$; while for $W<W_{c}$ it grows with $L$, indicating a divergence in the thermodynamic limit. The critical $W_{c}$ subject to error bars is the grey shaded region, estimated by positing $y_{0, \text { typ }}=A+B N^{\beta} ; \beta=0$ implies a localized phase and its deviation from 0 the onset of delocalization. (b) Color map of $\beta$ as a function of $(W, \omega)$. The blue line shows the contour $\beta=0.05$ as an estimate of the critical line (mobility edges); the value is chosen in accordance with the error bars in $\beta$. For $\omega=0$ our best estimate is $W_{c}(\omega=0) \simeq 6.8$. (c) Distribution of $y_{0}^{[L]}$ in the localized phase. Data are well converged for different $L$, and in excellent agreement with a Lévy distribution, $\sqrt{\kappa / \pi} y_{0}^{-3 / 2} e^{-\kappa / y_{0}}$ (with $\kappa=3.1 \times 10^{-2}$ ), shown by the grey shaded region. Statistics are obtained over $5 \times 10^{4}$ disorder realizations.

$$
\begin{aligned}
& y_{0}=\sum_{l=1}^{\infty} \phi_{l}, \\
& \phi_{l}=\sum_{i_{1} \in \mathrm{N}[0]} \frac{\Gamma^{2}}{\Omega_{i_{1}}^{2}} \sum_{i_{2} \in \mathrm{N}\left[i_{1}\right]} \frac{\Gamma^{2}}{\Omega_{i_{2}}^{2}} \cdots \sum_{i_{l} \in \mathrm{N}\left[i_{l-1}\right]} \frac{\Gamma^{2}}{\Omega_{i_{l}}^{2}},
\end{aligned}
$$

with $\phi_{l}$ the total contribution to $y_{0}$ from all sites on the $l$ th generation. Diagrammatically, it is the total contribution to $y_{0}$ from all $K^{l}$ paths of length $2 l$, each of which goes from the root site to a unique site in the $l$ th generation and retraces itself back to the root site [56]. For the series in Eq. (4) to converge in the thermodynamic limit, $\phi_{l}$ must decrease sufficiently fast with increasing $l$. This suggests that the distributions $P_{\phi_{l}}$ of $\phi_{l}$, should evolve with $l$ in a qualitatively different manner in the delocalized and localized phases. Calculating $P_{\phi_{1}}$ shows that this is indeed so, as shown in Figs. 2(a)-2(b). For strong disorder (localized phase), the vast bulk of the distribution shifts rapidly to smaller values with increasing $l$, while in the delocalized phase the support of the $P_{\phi_{l}}$ moves to larger values with increasing $l$. This is itself indicative of the convergence of the series in the localized phase and otherwise in the delocalized. To further quantify the convergence, one can define $y_{0}^{[l]} \equiv \sum_{n=1}^{l} \phi_{n}$ and study its typical value, $y_{0, \text { typ }}^{[l]}$, as a function of $l$ and $W$. Representative results at $\omega=0$ are shown in Fig. 2(c). For weak disorder, $y_{0, \text { typ }}^{[l]}$ grows rapidly with $l$, whereas for strong disorder it saturates to its converged value in the localized phase; again clearly showing the presence of a localization transition.

Two further remarks should be made. First, the recursive formulation also treats the real parts of all self-energies exactly. One can however make the simplifying approximation of neglecting them-Anderson's "upper limit approximation" [1,32]. For the tree with correlated disorder this approximation again predicts the presence of a transition, albeit naturally at a higher $W_{c}$ [51]. Second, the terms appearing in the series in Eq. (7) but with $X_{i_{n}}^{\left(i_{n-1}\right)}=0$ (i.e., $\Omega_{i_{n}} \equiv \omega-W \epsilon_{i_{n}}$ ) are precisely those appearing in the forward approximation [20]. By including the contribution of nonlocal propagators to the local propagator in an exact, fully renormalized fashion, the recursive formulation is a significant technical advance.
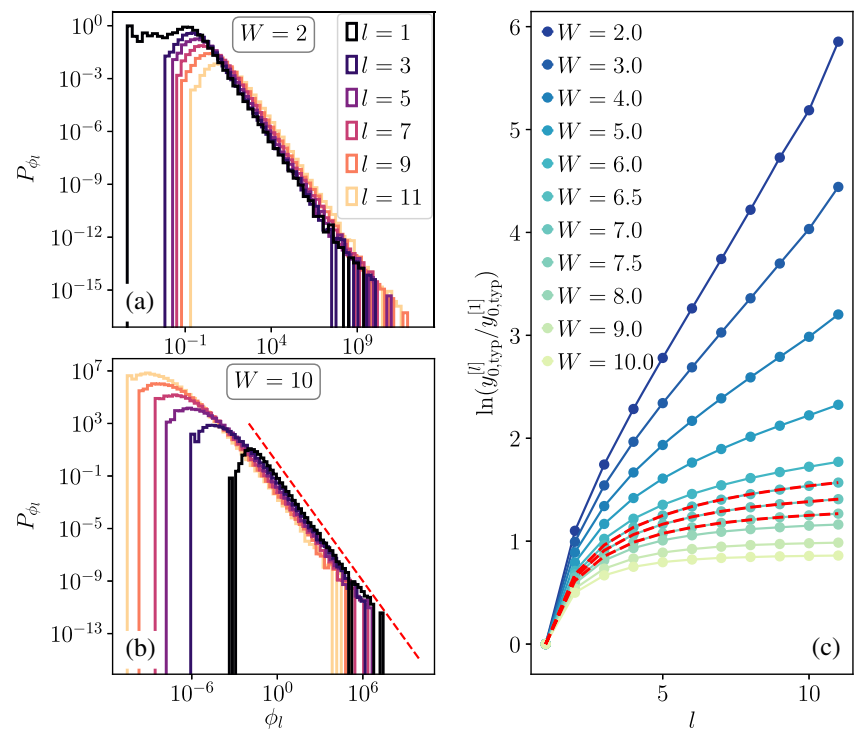

FIG. 2. Convergence of the series for $y_{0}$ [Eq. (7)] for the $K=2$ Cayley tree. (a),(b) Distributions $P_{\phi_{l}}$ in delocalized and localized phases, for different $l$; evolution with $l$ is qualitatively different in the two phases. Dashed line for localized phase shows Lévy tail, slope $-\frac{3}{2}$. (c) The typical value of the series summed to $l$ terms (normalized by the $l=1$ value) vs $l$. Results for $L=12, \omega=0$, and $5 \times 10^{4}$ realizations. Red lines show $W$ values lying in the critical regime shown in Fig. 1(a). 
Correlations in the $\epsilon_{i}$ 's preclude an exact analytic solution for the distribution of $y_{0}$ from Eq. (5). One can nevertheless perform a self-consistent mean-field calculation analytically at leading order in the renormalized perturbation series $[21,24,27]$ (here illustrated for $\omega=0$ ). Here $y_{0}$ depends only on the site energies of its neighbors, $\left\{i_{1}\right\}$. Since $\ell_{0 i_{1}}=1$, the maximally correlated limit implies the conditional distribution $P\left(\epsilon_{i_{1}} \mid \epsilon_{0}\right)=$ $\delta\left(\epsilon_{i_{1}}-\epsilon_{0}\right)$ in the thermodynamic limit. The distribution of $y_{0}$ can thus be simply calculated as $P_{y_{0}}\left(y_{0}\right)=$ $\int d \epsilon_{0} P\left(\epsilon_{0}\right) \delta\left(y_{0}-K \Gamma^{2}\left(1+y_{0, \text { typ }}\right) /\left[W^{2} \epsilon_{0}^{2}\right]\right)$. Since the univariate distribution $P\left(\epsilon_{0}\right)$ is a standard normal, this yields $P_{y_{0}}\left(y_{0}, y_{0, \text { typ }}\right)=\sqrt{\kappa / \pi} e^{-\kappa / y_{0}} y_{0}^{-3 / 2}$ where $\kappa=$ $K\left(1+y_{0, \text { typ }}\right) \Gamma^{2} / 2 W^{2}$. Remarkably and reassuringly, the distribution indeed has the Lévy form, just as obtained numerically by summing the entire series [Fig. 1(c)].

Self-consistency can now be imposed by requiring $\ln y_{0, \text { typ }}=\int d y_{0} P_{y_{0}}\left(y_{0}, y_{0, \text { typ }}\right) \ln y_{0}$; the solution of which is $y_{0, \text { typ }}=2 e^{\gamma} K \Gamma^{2}\left(W^{2}-2 e^{\gamma} K \Gamma^{2}\right)^{-1}$, with $\gamma$ the EulerMascheroni constant. Since $y_{0}$ is necessarily non-negative, self-consistency of the localized phase requires $W \geq W_{c}$, with [57]

$$
W_{c}=\sqrt{2} e^{\gamma / 2} \Gamma \sqrt{K}
$$

This $W_{c} \propto \sqrt{K}$ scaling is qualitatively different from that arising for uncorrelated disorder, where $W_{c} \propto K \ln K$ [32]; and stems intrinsically from the maximal correlations in the disorder.

We turn now to results arising for RRGs, via exact diagonalization (ED) of tight-binding Hamiltonians Eq. (1) with maximally correlated disorder Eq. (2). Our motivation here is twofold. First, while results above were for a rooted Cayley tree, we expect them to hold qualitatively for other random graphs. Second, it is important to corroborate the results with other independent measures of localization. Cayley trees are not moreover readily amendable to ED, since a finite fraction of sites live on the boundary; this issue is sidestepped by considering RRGs, which are locally treelike but contain long loops.

In the following we consider RRGs with a coordination number $Z=K+1=3$; denoting the total number of sites in the RRG by $N$. In accordance with the form of the covariance matrix for the Cayley tree, we take $C_{i j}=\exp \left[-\ell_{i j} \ln K / \ln N\right]$. The quantities studied will be the level spacing ratios, and $\Delta_{i}$ computed directly. We focus on the middle of the spectrum $(\omega=0)$ and consider 50-100 eigenstates therein.

For an ordered set of eigenvalues $\left\{E_{n}\right\}$, the level spacing ratio is $r_{n}=\min \left[s_{n}, s_{n+1}\right] / \max \left[s_{n}, s_{n+1}\right]$ with $s_{n}=E_{n}-E_{n-1}$. In an ergodic phase the distribution of $r_{n}$ follows the Wigner-Dyson surmise with mean $\bar{r} \simeq 0.53$, while in a localized phase the distribution is Poisson with $\bar{r} \simeq 0.386$. Results for $\bar{r}$ vs $W$ are shown in Fig. 3(a), and
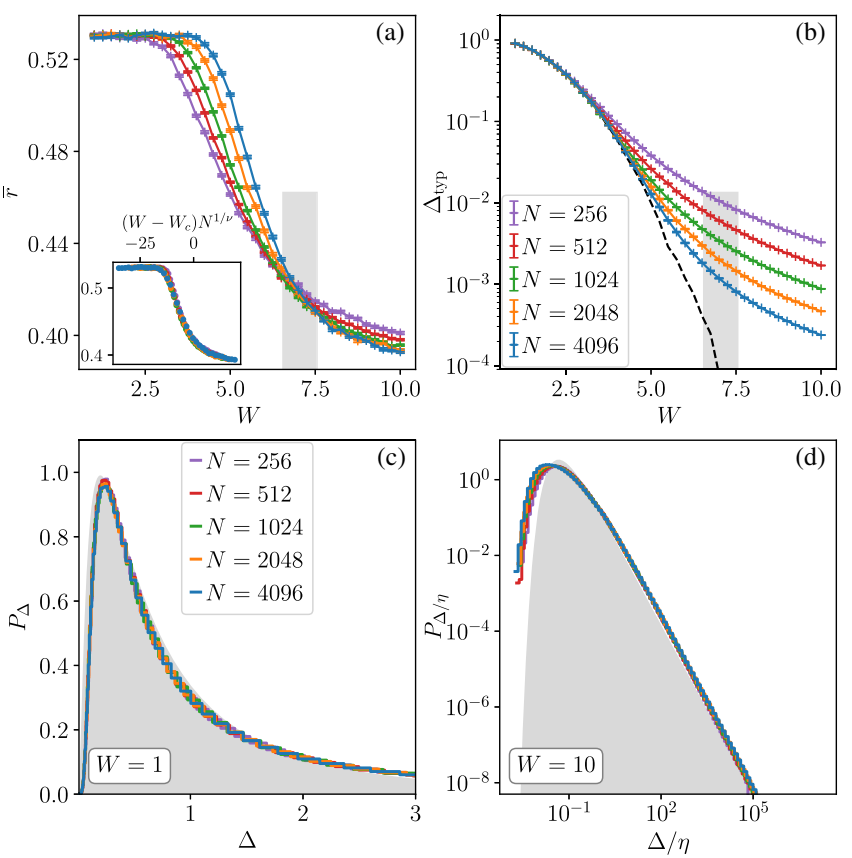

FIG. 3. ED results for a $K=2$ RRG with maximally correlated disorder. (a) Mean level spacing ratio vs $W$ shows a crossing for different $N$. Data collapse onto a common function of $\left(W-W_{c}\right) N^{1 / \nu}$ yields $W_{c} \simeq 6.8$ and $\nu \simeq 4.6$ (inset). (b) Typical value $\Delta_{\text {typ }}(\omega=0)$ computed exactly from Eq. (9). In the delocalized [localized] phase it is independent of [decays with] $N$. Dashed line shows extrapolation to $N \rightarrow \infty$. Grey shaded regions in (a), (b) denote the estimated critical region. (c),(d) Distributions of $\Delta$ and $y=\Delta / \eta$ in the delocalized and localized phases respectively. Grey shaded regions show best fits to lognormal and Lévy distributions, respectively.

show clearly a localization transition. A scaling collapse of the data for various $N$ onto a common function of $\left(W-W_{c}\right) N^{1 / \nu}$ yields a critical disorder strength of $W_{c} \simeq 6.8$ and $\nu \simeq 4.6$. Note that the $W_{c}$ estimated is remarkably close to that obtained above numerically for the $K=2$ Cayley tree.

From the set of exact eigenvalues $\left\{E_{n}\right\}$ and eigenstates $\left\{\left|\psi_{n}\right\rangle\right\}, \Delta_{i}(\omega)$ can be computed as

$$
\Delta_{i}(\omega)=\operatorname{Im}\left[G_{i}^{-1}(\omega)\right]-\eta, \quad G_{i}=\sum_{n} \frac{\left|\left\langle\psi_{n} \mid i\right\rangle\right|^{2}}{\omega+i \eta-E_{n}} .
$$

As $\Delta$ is finite with unit probability in the delocalized phase, $\Delta_{\text {typ }}$ should converge to a finite value with increasing $N$; while in a localized phase $\Delta \propto \eta$ vanishes with unit probability, so $\Delta_{\text {typ }}$ should decrease with $N$. This behavior is indeed found, see Fig. 3(b). To estimate numerically the critical $W_{c}$, we posit $\Delta_{\text {typ }}=\Delta_{\text {typ }, N \rightarrow \infty}+a / N^{\beta}$ and extrapolate the data to the thermodynamic limit. As shown in Fig. 3(b), the vanishing of $\Delta_{\text {typ }, N \rightarrow \infty}$ gives a $W_{c}$ consistent with that obtained from level statistics. In the localized phase, the distribution of $y=\Delta / \eta$ is again in very good 
agreement with a Lévy distribution [see Fig. 3(d)]. In the delocalized phase by contrast, $\Delta$ is qualitatively different, and appears to be log-normally distributed [Fig. 3(c)].

As above, whether for a Cayley tree or RRG, we find a one-parameter Lévy distribution for $y=\Delta / \eta$ in the localized phase. Importantly, it is thus universal: distributions for different $W>W_{c}$ can be collapsed onto a universal form by scaling the self-energy as $y / y_{\text {typ }}$ [51]. Further, the distribution can be directly connected to that of wave function amplitudes, the moments of which [via generalized inverse participation ratios (IPRs)] probe the divergence of the localization length, $\xi$, as $W \rightarrow W_{c}$ [51]. Within our mean-field theory, we find $\xi \sim\left(W-W_{c}\right)^{-1}$ with an exponent of 1 .

We turn now to the $K \rightarrow \infty$ limit. For any one-body problem to remain well defined in this limit, the hopping must be rescaled as $\Gamma=\Gamma_{*} / \sqrt{K}$ [58]. The mean-field theory then yields a finite critical $W_{c}=\sqrt{2} e^{\gamma / 2} \Gamma_{*}$; in stark contrast to the case of uncorrelated disorder where, despite rescaling $\Gamma, W_{c} / \Gamma_{*} \propto \sqrt{K} \ln K$ thus precludes localization as $K \rightarrow \infty$. For MBL on Fock space, in a system containing $L$ real-space sites, the effective connectivity on the Fock-space graph scales as $K \sim L$, and the effective Fock-space disorder as $W_{\mathrm{FS}} \sim \sqrt{L} W_{t}$ [with $W_{t} \sim \mathcal{O}(1)$ ] $[21,27]$. Rescaling all energies by $\sqrt{L}$, as required to attain a well-defined thermodynamic limit $L \rightarrow \infty$, again leads [27] to a finite critical $W_{t, c}$, in direct parallel to the $K \rightarrow \infty$ limit of the present problem. The existence of a MBL phase thus provides an indirect but complementary argument for the $\sqrt{K}$ scaling of $W_{c}$.

In summary, we have studied AL on Cayley trees and RRGs with maximally correlated on-site disorder, mirroring the effective Fock-space disorder of MBL systems. While such correlations might be thought to disfavor localization by suppressing site-energy fluctuations, we find both that an Anderson transition is present, and that scaling of the critical disorder with graph connectivity is qualitatively different from that of uncorrelated disorder, with correlations favoring localization. Our results address a new class of AL problems, and shed light on the crucial role played by correlations in Fock-space disorder in stabilizing MBL. Many questions arise as to what further aspects of MBL can be captured by $\mathrm{AL}$ problems with maximally correlated disorder. One such is the multifractal character of wave functions, and its possible connection to the anomalous statistics of MBL wave functions on Fock space; and our preliminary results indeed suggest the presence of multifractal eigenstates on RRGs. Looking further afield, understanding the effect of maximal correlations on glassy dynamics on such graphs is also immanently important.

We thank J. T. Chalker, A. Duthie, and A. Lazarides for useful discussions and comments on the manuscript. This work was in part supported by EPSRC Grant No. EP/ S020527/1. *sthitadhi.roy@chem.ox.ac.uk

†david.logan@chem.ox.ac.uk

[1] P. W. Anderson, Absence of diffusion in certain random lattices, Phys. Rev. 109, 1492 (1958).

[2] I. V. Gornyi, A. D. Mirlin, and D. G. Polyakov, Interacting Electrons in Disordered Wires: Anderson Localization and Low-T Transport, Phys. Rev. Lett. 95, 206603 (2005).

[3] D. M. Basko, I. L. Aleiner, and B. L. Altshuler, Metalinsulator transition in a weakly interacting many-electron system with localized single-particle states, Ann. Phys. (Amsterdam) 321, 1126 (2006).

[4] V. Oganesyan and D. A. Huse, Localization of interacting fermions at high temperature, Phys. Rev. B 75, 155111 (2007).

[5] A. Pal and D. A. Huse, Many-body localization phase transition, Phys. Rev. B 82, 174411 (2010).

[6] R. Nandkishore and D. A. Huse, Many-body localization and thermalization in quantum statistical mechanics, Annu. Rev. Condens. Matter Phys. 6, 15 (2015).

[7] F. Alet and N. Laflorencie, Many-body localization: An introduction and selected topics, C.R. Phys. 19, 498 (2018).

[8] D. A. Abanin, E. Altman, I. Bloch, and M. Serbyn, Colloquium: Many-body localization, thermalization, and entanglement, Rev. Mod. Phys. 91, 021001 (2019).

[9] J. A. Kjäll, J. H. Bardarson, and F. Pollmann, Many-Body Localization in a Disordered Quantum Ising Chain, Phys. Rev. Lett. 113, 107204 (2014).

[10] D. J. Luitz, N. Laflorencie, and F. Alet, Many-body localization edge in the random-field Heisenberg chain, Phys. Rev. B 91, 081103(R) (2015).

[11] R. Vosk, D. A. Huse, and E. Altman, Theory of the ManyBody Localization Transition in One-Dimensional Systems, Phys. Rev. X 5, 031032 (2015).

[12] A. C. Potter, R. Vasseur, and S. A. Parameswaran, Universal Properties of Many-Body Delocalization Transitions, Phys. Rev. X 5, 031033 (2015).

[13] A. Goremykina, R. Vasseur, and M. Serbyn, Analytically Solvable Renormalization Group for the Many-Body Localization Transition, Phys. Rev. Lett. 122, 040601 (2019).

[14] P. T. Dumitrescu, A. Goremykina, S. A. Parameswaran, M. Serbyn, and R. Vasseur, Kosterlitz-thouless scaling at manybody localization phase transitions, Phys. Rev. B 99, 094205 (2019).

[15] A. Morningstar and D. A. Huse, Renormalization-group study of the many-body localization transition in one dimension, Phys. Rev. B 99, 224205 (2019).

[16] A. Morningstar, D. A. Huse, and J. Z. Imbrie, Many-body localization near the critical point, Phys. Rev. B 102, 125134 (2020).

[17] D. E. Logan and P. G. Wolynes, Quantum localization and energy flow in many-dimensional fermi resonant systems, J. Chem. Phys. 93, 4994 (1990).

[18] B. L. Altshuler, Y. Gefen, A. Kamenev, and L. S. Levitov, Quasiparticle Lifetime in a Finite System: A Nonperturbative Approach, Phys. Rev. Lett. 78, 2803 (1997).

[19] C. Monthus and T. Garel, Many-body localization transition in a lattice model of interacting fermions: Statistics of renormalized hoppings in configuration space, Phys. Rev. B 81, 134202 (2010). 
[20] F. Pietracaprina, V. Ros, and A. Scardicchio, Forward approximation as a mean-field approximation for the Anderson and many-body localization transitions, Phys. Rev. B 93, 054201 (2016).

[21] D. E. Logan and S. Welsh, Many-body localization in Fock space: A local perspective, Phys. Rev. B 99, 045131 (2019).

[22] S. Roy, D. E. Logan, and J. T. Chalker, Exact solution of a percolation analog for the many-body localization transition, Phys. Rev. B 99, 220201(R) (2019).

[23] S. Roy, J. T. Chalker, and D. E. Logan, Percolation in fock space as a proxy for many-body localization, Phys. Rev. B 99, 104206 (2019).

[24] S. Roy and D. E. Logan, Self-consistent theory of manybody localisation in a quantum spin chain with long-range interactions, SciPost Phys. 7, 42 (2019).

[25] F. Pietracaprina and N. Laflorencie, Hilbert space fragmentation and many-body localization, arXiv:1906.05709.

[26] S. Ghosh, A. Acharya, S. Sahu, and S. Mukerjee, Manybody localization due to correlated disorder in fock space, Phys. Rev. B 99, 165131 (2019).

[27] S. Roy and D. E. Logan, Fock-space correlations and the origins of many-body localization, Phys. Rev. B 101, 134202 (2020).

[28] A. Croy, P. Cain, and M. Schreiber, Anderson localization in 1d systems with correlated disorder, Eur. Phys. J. B 82, 107 (2011).

[29] A. Croy, P. Cain, and M. Schreiber, The role of power-law correlated disorder in the Anderson metal-insulator transition, Eur. Phys. J. B 85, 165 (2012).

[30] E. Fratini and S. Pilati, Anderson localization in optical lattices with correlated disorder, Phys. Rev. A 92, 063621 (2015).

[31] P. A. Nosov, I. M. Khaymovich, and V. E. Kravtsov, Correlation-induced localization, Phys. Rev. B 99, 104203 (2019).

[32] R. Abou-Chacra, D. J. Thouless, and P. W. Anderson, A self-consistent theory of localization, J. Phys. C 6, 1734 (1973).

[33] J. T. Chalker and S. Siak, Anderson localisation on a Cayley tree: A new model with a simple solution, J. Phys. Condens. Matter 2, 2671 (1990).

[34] A. De Luca, B. L. Altshuler, V.E. Kravtsov, and A. Scardicchio, Anderson Localization on the Bethe Lattice: Nonergodicity of Extended States, Phys. Rev. Lett. 113, 046806 (2014).

[35] B. L. Altshuler, L. B. Ioffe, and V. E. Kravtsov, Multifractal states in self-consistent theory of localization: Analytical solution, arXiv:1610.00758.

[36] K. S. Tikhonov, A. D. Mirlin, and M. A. Skvortsov, Anderson localization and ergodicity on random regular graphs, Phys. Rev. B 94, 220203(R) (2016).

[37] I. García-Mata, O. Giraud, B. Georgeot, J. Martin, R. Dubertrand, and G. Lemarié, Scaling Theory of the Anderson Transition in Random Graphs: Ergodicity and Universality, Phys. Rev. Lett. 118, 166801 (2017).

[38] M. Sonner, K. S. Tikhonov, and A. D. Mirlin, Multifractality of wave functions on a Cayley tree: From root to leaves, Phys. Rev. B 96, 214204 (2017).

[39] G. Biroli and M. Tarzia, Delocalization and ergodicity of the Anderson model on Bethe lattices, arXiv:1810.07545.
[40] V. E. Kravtsov, B. L. Altshuler, and L. B. Ioffe, Non-ergodic delocalized phase in anderson model on bethe lattice and regular graph, Ann. Phys. (Amsterdam) 389, 148 (2018).

[41] K. S. Tikhonov and A. D. Mirlin, Critical behavior at the localization transition on random regular graphs, Phys. Rev. B 99, 214202 (2019).

[42] S. Savitz, C. Peng, and G. Refael, Anderson localization on the Bethe lattice using cages and the Wegner flow, Phys. Rev. B 100, 094201 (2019).

[43] G. Parisi, S. Pascazio, F. Pietracaprina, V. Ros, and A. Scardicchiozio, Anderson transition on the Bethe lattice: an approach with real energies, J. Phys. A 53014003 (2020).

[44] I. García-Mata, J. Martin, R. Dubertrand, O. Giraud, B. Georgeot, and G. Lemarié, Two critical localization lengths in the Anderson transition on random graphs, Phys. Rev. Research 2, 012020 (2020).

[45] M. Tarzia, The many-body localization transition in the Hilbert space, Phys. Rev. B 102, 014208 (2020).

[46] G. Biroli and M. Tarzia, Delocalized glassy dynamics and many-body localization, Phys. Rev. B 96, 201114(R) (2017).

[47] G. Biroli and M. Tarzia, Anomalous dynamics in the ergodic side of the many-body localization transition and the glassy phase of directed polymers in random media, Phys. Rev. B 102, 064211 (2020).

[48] G. De Tomasi, S. Bera, A. Scardicchio, and I. M. Khaymovich, Subdiffusion in the Anderson model on the random regular graph, Phys. Rev. B 101, 100201(R) (2020).

[49] S. Welsh and D. E. Logan, Simple probability distributions on a Fock-space lattice, J. Phys. Condens. Matter 30, 405601 (2018).

[50] The algorithm for constructing the correlated energies is described in the Supplemental Material [51].

[51] See Supplemental Material at http://link.aps.org/supplemental/ 10.1103/PhysRevLett.125.250402 for details on (i) the upperlimit approximation, (ii) relation between wave function densities and localisation length, (iii) the construction of correlated energies, and (iv) mean-field mobility edges.

[52] E. N. Economou and M. H. Cohen, Existence of mobility edges in Anderson's model for random lattices, Phys. Rev. B 5, 2931 (1972).

[53] D. J. Thouless, Electrons in disordered systems and the theory of localization, Phys. Rep. 13, 93 (1974).

[54] D. C. Licciardello and E. N. Economou, Study of localization in Anderson's model for random lattices, Phys. Rev. B 11, 3697 (1975).

[55] D. E. Logan and P. G. Wolynes, Anderson localization in topologically disordered systems, Phys. Rev. B 31, 2437 (1985); D. E. Logan and P. G. Wolynes, Dephasing and anderson localization in topologically disordered systems, Phys. Rev. B 36, 4135 (1987).

[56] On a tree, there exists a unique shortest path between any pair of sites. For a site on generation $l$, the length of the corresponding path between it and the root site is $l$.

[57] While this analysis focuses on the localized phase, selfconsistency for the delocalized phase commensurately breaks down at the same $W_{c}$ as in Eq. (8) [21,24].

[58] A. Georges, G. Kotliar, W. Krauth, and M. J. Rozenberg, Dynamical mean-field theory of strongly correlated fermion systems and the limit of infinite dimensions, Rev. Mod. Phys. 68, 13 (1996). 